\title{
Theoretical Study of Ultra-Relativistic Laser Electron Interaction in the Strong Radiation Reaction Regime*)
}

\author{
Keita SETO, Hideo NAGATOMO and Kunioki MIMA \\ Institute of Laser Engineering, Osaka University, 2-6 Yamada-oka, Suita 565-0871, Japan
}

(Received 6 December 2010 / Accepted 11 March 2011)

\begin{abstract}
In the near future, the intensity of the ultra-short pulse laser will reach to $10^{22} \mathrm{~W} / \mathrm{cm}^{2}$ owe to the advancements of the laser technologies. The motion of the electron becomes relativism. If the electron is laid in such strong field, the effect of the "radiation reaction" is not negligible for electron motion. In general, if this motion is describe as the Lorentz-Abraham-Dirac equation, there is a "run-away" solution. A lot of researchers have tried to transform this equation for avoiding run-away. As one solution of this problem, we succeeded in the discovery of a new equation that takes the place of the Lorentz-Abraham-Dirac equation. I'll show the validity of this equation by using the simulation in this paper.
\end{abstract}

(c) 2011 The Japan Society of Plasma Science and Nuclear Fusion Research

Keywords: radiation reaction, reaction force, laser electron interaction, exawatt laser, LAD equation, Schott force

DOI: $10.1585 /$ pfr.6.2404099

\section{Introduction}

With the rapid progress of the ultra-short pulse laser technologies, its intensity will reach to $10^{22} \mathrm{~W} / \mathrm{cm}^{2}$ [1]. If an electron is in the strong fields caused by the laser of which the intensity is larger than $10^{18} \mathrm{~W} / \mathrm{cm}^{2}$, the dynamics of the electron should be described by relativistic equations. The most important phenomenon in this regime is an effect of ponderomotive force, and an electron is pushed out in the propagation direction of laser. If the laser intensity is higher than $10^{22} \mathrm{~W} / \mathrm{cm}^{2}$, the strong bremsstrahlung might be caused. Simultaneously with it, the "radiation reaction force (or damping force)" works to charged particles. We can consider this effect as a "selfinteraction". In the laser electron interaction in this intensity level, one of the important equation is an equation of motion. In this dynamics, an equation of motion can be described as the Lorentz-Abraham-Dirac (LAD) equation in Minkowski spacetime [2].

$$
\begin{aligned}
& m_{0} \frac{\mathrm{d}}{\mathrm{d} \tau} w^{\mu}=Q F_{\text {laser }}^{\mu \nu} w_{v}+f_{\text {reaction }}^{\mu}, \\
& f_{\text {reaction }}^{\mu}=m_{0} \tau_{0} \frac{\mathrm{d}^{2}}{\mathrm{~d} \tau^{2}} w^{\mu}+\frac{m_{0} \tau_{0}}{c^{2}} g\left(\frac{\mathrm{d} w}{\mathrm{~d} \tau}, \frac{\mathrm{d} w}{\mathrm{~d} \tau}\right) w^{\mu} .
\end{aligned}
$$

Where $\tau_{0}=Q^{2} / 6 \pi \varepsilon_{0} m_{0} c^{3}, \tau$ is a proper time, $w$ is a 4 velocity defined as $w \equiv \gamma(c, \boldsymbol{v})$, and $g$ is Lorentz metric. Our metric signature is (+---). $\quad F_{\text {laser }}$ is an electromagnetic field tensor. In these equations, the force $f_{\text {reaction }}$ is called the "reaction force" or "damping force". This reaction force is generated from the energy loss of bremsstrahlung. This energy loss is described as

\footnotetext{
author'se-mail: seto-k@ile.osaka-u.ac.jp

*) This article is based on the presentation at the 20th International Toki Conference (ITC20).
}

$$
\frac{\mathrm{d} W}{\mathrm{~d} t}=-m_{0} \tau_{0} g\left(\frac{\mathrm{d} w}{\mathrm{~d} \tau}, \frac{\mathrm{d} w}{\mathrm{~d} \tau}\right),
$$

in the covariant form. The first term of Eq. (2) is called the "Schott force",

$$
f_{\text {Schott }} \equiv m_{0} \tau_{0} \frac{\mathrm{d}^{2} w}{\mathrm{~d} \tau^{2}}
$$

and the second term is the direct effect of the radiation. The solution of Eq. (1) is

$$
\begin{aligned}
\frac{\mathrm{d}}{\mathrm{d} \tau} w^{\mu}(\tau)= & \frac{\mathrm{d}}{\mathrm{d} \tau} w^{\mu}(0) \times e^{\frac{\tau}{\tau_{0}}} \\
& -\int_{0}^{\tau} \mathrm{d} \tau^{\prime}\left[\begin{array}{c}
\frac{Q}{m_{0} \tau_{0}} F_{\text {laser }}^{\mu \nu} w_{v} \\
+\frac{1}{c^{2}} g\left(\frac{\mathrm{d} w}{\mathrm{~d} \tau}, \frac{\mathrm{d} w}{\mathrm{~d} \tau}\right) w^{\mu}
\end{array}\right] e^{\frac{\tau-\tau^{\prime}}{\tau_{0}}} .
\end{aligned}
$$

Since $\tau_{0}$ is too small, the factor of $\exp \left(\tau / \tau_{0}\right)$ diverges to infinity at a moment. The first term of Eq. (5) generates infinite acceleration. This term is called as a "run-away" solution and it should be avoided because this motion isn't physical. One of the methods avoided a run-away, there is an equation of Landau-Lifshitz [3]. They suggested the second order derivative (Schott force) should be described by only external forces. The solution doesn't have a runaway, but it is only when the reaction force is smaller than external forces that we can apply this.

The reason why the run-away solution of the LAD equation cannot be avoid, is the equation of motion has a Schott term. When we think about zero (time) component of Eq. (1), it is described as follows.

$$
\frac{\mathrm{d} E}{\mathrm{~d} \tau}=\gamma Q \boldsymbol{E}_{\text {laser }} \cdot \boldsymbol{v}-\frac{\mathrm{d} W}{\mathrm{~d} \tau}+c f_{\text {Schott }}^{0} .
$$

This Eq. (6) means the change in the kinetic energy is equal to the work done by the external field minus the radiation 
plus something else, the Schott term.

The studies of R. T. Hammond suggest that the energy balance should be satisfied without the Schott term $[4,5]$. He assumes that the reaction force can be written as a gradient of the potential, and vanishes the Schott force from the zero component of the LAD equation. His potential method is one of great solution, but space components of the equation of motion become too difficult. Moreover, the assumption that the reaction force is a conservative is a strong condition. We will lead the equation of motion without the assumption of a conservative force, but we'll assume that the zero element of Hammond's equation is correct. And we'll simulate the motion by using our new equation.

\section{New Equation of Motion}

In this chapter, we'll lead a new equation of motion satisfied with the zero component of Hammond's equation. In other words, we want to search for space components of an equation without a gradient of a potential. The starting line of our discussion is an energy balance between a bremsstrahlung and work by a reaction force $\boldsymbol{F}_{\text {reaction }}$.

$$
\boldsymbol{F}_{\text {reaction }} \cdot \boldsymbol{v}+\frac{\mathrm{d} W}{\mathrm{~d} t}=0
$$

In a Minkowski spacetime, we use the zero component of a 4-reaction force,

$$
\begin{aligned}
f_{\text {reaction }}^{0} & =-\frac{\gamma}{c} \frac{\mathrm{d} W}{\mathrm{~d} t} \\
& =\frac{m_{0} \tau_{0}}{c^{2}} g\left(\frac{\mathrm{d} w}{\mathrm{~d} \tau}, \frac{\mathrm{d} w}{\mathrm{~d} \tau}\right) w^{0} .
\end{aligned}
$$

Here, $f_{\text {reaction }}^{0}=\gamma \boldsymbol{F}_{\text {reaction }} \cdot \boldsymbol{v} / c$ should be noted. This Eq. (8) means an energy loss by a bremsstrahlung. The total energy change of a particle is

$$
m_{0} \frac{\mathrm{d} w^{0}}{\mathrm{~d} \tau}=Q F_{\text {laser }}^{0 v} w_{v}+f_{\text {reaction }}^{0} .
$$

This is the equation which is suggested by Hammond. We'll derive the space components of the reaction force. Using Eq. (7) again, $f_{\text {reaction }}^{i=1,2,3}=\gamma \boldsymbol{F}_{\text {reaction, }}$,

$$
\sum_{i=1,2,3} \gamma^{-2} f_{\text {reaction }}^{i} w^{i}+\frac{\mathrm{d} W}{\mathrm{~d} t}=0 .
$$

Where, $i=1,2,3$ mean space components. This Eq. (10) transforms into Eq. (11).

$$
\begin{gathered}
\sum_{i=1,2,3} w^{i}\left[\begin{array}{c}
f_{\text {reaction }}^{i}-m_{0} \tau_{0} \frac{\mathrm{d}^{2} w^{i}}{\mathrm{~d} \tau^{2}} \\
-\frac{m_{0} \tau_{0}}{c^{2}} g\left(\frac{\mathrm{d} w}{\mathrm{~d} \tau}, \frac{\mathrm{d} w}{\mathrm{~d} \tau}\right) w^{i}
\end{array}\right] \\
+m_{0} \tau_{0} w^{0} \frac{\mathrm{d}^{2} w^{0}}{\mathrm{~d} \tau^{2}}=0 .
\end{gathered}
$$

In the case $\boldsymbol{v} \neq \mathbf{0}$, Eq. (11) is deformed as follow.

$$
\begin{aligned}
\frac{\mathrm{d}^{2} w^{0}}{\mathrm{~d} \tau^{2}} & =\sum_{i=1,2,3}\left(\frac{1}{\beta^{2}} \frac{\mathrm{d}^{2} w^{0}}{\mathrm{~d} \tau^{2}} \beta^{i}\right) \beta^{i} \\
& =\sum_{i=1,2,3}\left[\frac{1}{\left(w^{0}\right)^{2}-c^{2}} \frac{\mathrm{d}^{2} w^{0}}{\mathrm{~d} \tau^{2}} w^{i}\right] w^{i} .
\end{aligned}
$$

Using Eq. (12), Eq. (11) becomes

$$
\sum_{i=1,2,3} w^{i}\left[\begin{array}{c}
f_{\text {reaction }}^{i}-m_{0} \tau_{0} \frac{\mathrm{d}^{2} w^{i}}{\mathrm{~d} \tau^{2}} \\
-\frac{m_{0} \tau_{0}}{c^{2}} g\left(\frac{\mathrm{d} w}{\mathrm{~d} \tau}, \frac{\mathrm{d} w}{\mathrm{~d} \tau}\right) w^{i} \\
+m_{0} \tau_{0} \frac{w^{0}}{\left(w^{0}\right)^{2}-c^{2}} \frac{\mathrm{d}^{2} w^{0}}{\mathrm{~d} \tau^{2}} w^{i}
\end{array}\right]=0
$$

Hence the 4-velocity is arbitrary in generally,

$$
\begin{aligned}
f_{\text {reaction }}^{i}= & m_{0} \tau_{0} \frac{\mathrm{d}^{2} w^{i}}{\mathrm{~d} \tau^{2}} \\
& +\frac{m_{0} \tau_{0}}{c^{2}} g\left(\frac{\mathrm{d} w}{\mathrm{~d} \tau}, \frac{\mathrm{d} w}{\mathrm{~d} \tau}\right) w^{i} \\
& -m_{0} \tau_{0} \frac{w^{0}}{\left(w^{0}\right)^{2}-c^{2}} \frac{\mathrm{d}^{2} w^{0}}{\mathrm{~d} \tau^{2}} w^{i} .
\end{aligned}
$$

Next, we need to think NON-relativistic region; $\beta \rightarrow 0$ and $\gamma \rightarrow 1$. In this region, you know, the reaction force becomes the Schott force [3].

$$
\lim _{\beta \rightarrow 0} f_{\text {reaction }}^{i}=m_{0} \tau_{0} \frac{\mathrm{d}^{2} w^{i}}{\mathrm{~d} \tau^{2}}=f_{\text {Schott }}^{i} .
$$

The second term of Eq. (14) becomes zero, but the third term has a possibility of divergence. Therefore we take a small value of $\varepsilon$ for avoiding a singular point, and rewrite from Eq. (14) to

$$
\begin{aligned}
f_{\text {reaction }}^{i}= & m_{0} \tau_{0} \frac{\mathrm{d}^{2} w^{i}}{\mathrm{~d} \tau^{2}} \\
& +\frac{m_{0} \tau_{0}}{c^{2}} g\left(\frac{\mathrm{d} w}{\mathrm{~d} \tau}, \frac{\mathrm{d} w}{\mathrm{~d} \tau}\right) w^{i} \\
& -m_{0} \tau_{0} \frac{w^{0}}{\left(w^{0}\right)^{2}-c^{2}+\left|\varepsilon_{\rightarrow 0}\right|} \frac{\mathrm{d}^{2} w^{0}}{\mathrm{~d} \tau^{2}} w^{i} .
\end{aligned}
$$

Where, the manner is introduced as

$$
f\left(\varepsilon_{\rightarrow 0}\right) \equiv \lim _{\varepsilon \rightarrow 0} f(\varepsilon) .
$$

Finally, let's unite time and space components of the reaction force.

$$
\begin{aligned}
f_{\text {reaction }}^{\mu}= & m_{0} \tau_{0} \frac{\mathrm{d}^{2} w^{\mu}}{\mathrm{d} \tau^{2}} \\
& +\frac{m_{0} \tau_{0}}{c^{2}} g\left(\frac{\mathrm{d} w}{\mathrm{~d} \tau}, \frac{\mathrm{d} w}{\mathrm{~d} \tau}\right) w^{\mu} \\
& -m_{0} \tau_{0} \frac{w^{0} w^{\mu}-c^{2} \delta_{0}^{\mu}}{\left(w^{0}\right)^{2}-c^{2}+\left|\varepsilon_{\rightarrow 0}\right|} \frac{\mathrm{d}^{2} w^{0}}{\mathrm{~d} \tau^{2}}
\end{aligned}
$$

Of course, this equation fulfills the relativistic invariant.

$$
g\left(f_{\text {reaction }}, w\right)=0 .
$$

Therefore, the equation of motion in an electromagnetic field becomes as follows.

$$
\begin{aligned}
m_{0} \frac{\mathrm{d} w^{\mu}}{\mathrm{d} \tau}= & Q F_{\text {laser }}^{\mu v} w_{v}+m_{0} \tau_{0} \frac{\mathrm{d}^{2} w^{\mu}}{\mathrm{d} \tau^{2}} \\
& +\frac{m_{0} \tau_{0}}{c^{2}} g\left(\frac{\mathrm{d} w}{\mathrm{~d} \tau}, \frac{\mathrm{d} w}{\mathrm{~d} \tau}\right) w^{\mu} \\
& -m_{0} \tau_{0} \frac{w^{0} w^{\mu}-c^{2} \delta_{0}^{\mu}}{\left(w^{0}\right)^{2}-c^{2}+\left|\varepsilon_{\rightarrow 0}\right|} \frac{\mathrm{d}^{2} w^{0}}{\mathrm{~d} \tau^{2}} .
\end{aligned}
$$


This equation cannot have a run-away solution. The zero component of Eq. (20) or Eq. (9) can be solved strictly.

$$
\frac{\mathrm{d} w^{0}}{\mathrm{~d} \tau}+\frac{1}{m_{0} c^{2}} \frac{\mathrm{d} W}{\mathrm{~d} t} w^{0}=\frac{Q}{m_{0}} F_{\text {laser }}^{0 v} w_{v} .
$$

This Eq. (21) is a very basic, first-linear ordinary differential equation. Therefore, the solution is,

$$
\begin{aligned}
w^{0}(\tau)= & w^{0}(0) \times \exp \left[-\int_{0}^{\tau} \mathrm{d} \tau^{\prime} \frac{1}{m_{0} c^{2}} \frac{\mathrm{d} W}{\mathrm{~d} t}\left(\tau^{\prime}\right)\right] \\
& +\int_{0}^{\tau} \mathrm{d} \tau^{\prime} \frac{Q}{m_{0}} F_{\text {laser }}^{0 v} w_{v}\left(\tau^{\prime}\right) \\
& \times \exp \left[-\int_{\tau^{\prime}}^{\tau} \mathrm{d} \tau^{\prime \prime} \frac{1}{m_{0} c^{2}} \frac{\mathrm{d} W}{\mathrm{~d} t}\left(\tau^{\prime \prime}\right)\right]
\end{aligned}
$$

A bremsstrahlung energy loss $\mathrm{d} W / \mathrm{d} t$ can't become negative, the solution Eq. (22) free from a run-away effect. Other space components are connected by the relation which $g(w, w)=c^{2}$. If one of the $w^{i=1,2,3}$ become run-away, the relativistic relation $g(w, w)=c^{2}$ cannot be satisfied. Therefore space components cannot become a run-away state. From the above, it becomes a conclusion which the solution of Eq. (20) does not have a run-away factor.

\section{Configurations and Condition for Simulation}

Next, we researched the numerical calculations for the characteristic of Eq. (20). The purpose of these simulations is check of the radiation reaction effects. We assume that a charged particle is an electron, $Q \mapsto-e$ and $m_{0}$ is a rest mass of an electron. Our simulations are very basic firstorder leap-flog situations of laser electron interactions.

The first configuration is initial stationary condition (see Fig. 1). An initial stationary electron is irradiated by the Gaussian and linear polarization laser pulse which has an intensity of $5 \times 10^{22} \mathrm{~W} / \mathrm{cm}^{2}$, a wave length of $1 \mu \mathrm{m}$ and a pulse width is $20 \mathrm{fsec}$. An electron is in $200 \times \lambda_{\text {laser }}$ from the peak place of the laser pulse. In our simulation, the propagating direction is $x$, the electric and magnetic field is excited $y$ and $z$ directions. Therefore the shape of electric field is

$$
\begin{aligned}
\boldsymbol{E}= & \hat{\boldsymbol{y}} E_{0} \exp \left[-\frac{\left(\omega_{\text {laser }} t-k_{\text {laser }} x\right)^{2}}{\left(\omega_{\text {laser }} \Delta t\right)^{2}}\right] \\
& \times \sin \left(\omega_{\text {laser }} t-k_{\text {laser }} x\right) .
\end{aligned}
$$

$\hat{\boldsymbol{y}}$ is an unit vector of $y$ direction. Since the external laser field is linear polarization,

$$
\begin{aligned}
\boldsymbol{B}= & \hat{z} \frac{E_{0}}{c} \exp \left[-\frac{\left(\omega_{\text {laser }} t-k_{\text {laser }} x\right)^{2}}{\left(\omega_{\text {laser }} \Delta t\right)^{2}}\right] \\
& \times \sin \left(\omega_{\text {laser }} t-k_{\text {laser }} x\right) .
\end{aligned}
$$

This configuration shows us the smoothly shift of the physical region from NON-relativism to relativism. The peak intensity of the laser pulse generates a relativistic motion of an electron, therefore it is important that we consider this configuration.

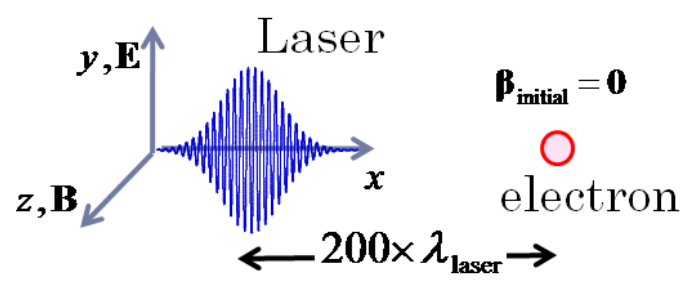

Fig. 1 Configuration of an initial stationary electron and an incident Gaussian laser pulse. This laser has an intensity of $5 \times 10^{22} \mathrm{~W} / \mathrm{cm}^{2}$, a wave length of $1 \mu \mathrm{m}$ and a pulse width is $20 \mathrm{fsec}$. An electron is in $200 \times \lambda_{\text {laser }}$ from the peak place of the laser pulse.

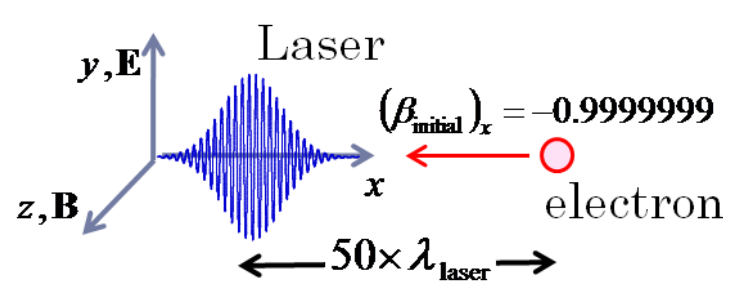

Fig. 2 Setting of counter configuration. This laser has an intensity of $5 \times 10^{22} \mathrm{~W} / \mathrm{cm}^{2}$, a wave length of $1 \mu \mathrm{m}$ and a pulse width is $20 \mathrm{fsec}$. Initially, an electron is in $50 \times \lambda_{\text {lambda }}$ from the peak place of the laser pulse and has an energy of $1 \mathrm{GeV}$.

The second configuration is that an electron with energy of $1 \mathrm{GeV}$ propagates to the counter direction of the laser (see Fig. 2). The parameters of laser are the same condition of the initial stationary electron. An electron is in $50 \times \lambda_{\text {laser }}$ from the peak place of the laser pulse and propagate $-x$ direction at initial. In this situation, the laser stop an electron motion, and then an electron lose a big energy as a bremsstrahlung. From Eq. (7), we know, a big reaction force will be generated. Therefore, this configuration is one of the best situations which is easy to observe a reaction effect.

\section{Simulation Result}

In this chapter, we'll show the simulation result of Eq. (20) on two situations. The first situation is an initial stationary condition. The trajectory in $x y$ space of the electron described as Fig. 3. There are two lines in this figure, the red one is a trajectory of an electron without a reaction force, the solution as follow equation.

$$
m_{0} \frac{\mathrm{d} w^{\mu}}{\mathrm{d} \tau}=Q F_{\text {laser }}^{\mu v} w_{v}
$$

And the blue one is the trajectory with a reaction force, the full solution of Eq. (20). Two lines are roughly the same trajectories. This means that there is little difference between the reaction-ON case and the reaction-OFF case. The directions of the velocity and acceleration turn in $x$ direction. If the main direction of the acceleration is the same of the velocity like this case, a reaction force effect becomes small. The time evolution of the energy $(\gamma)$ is 


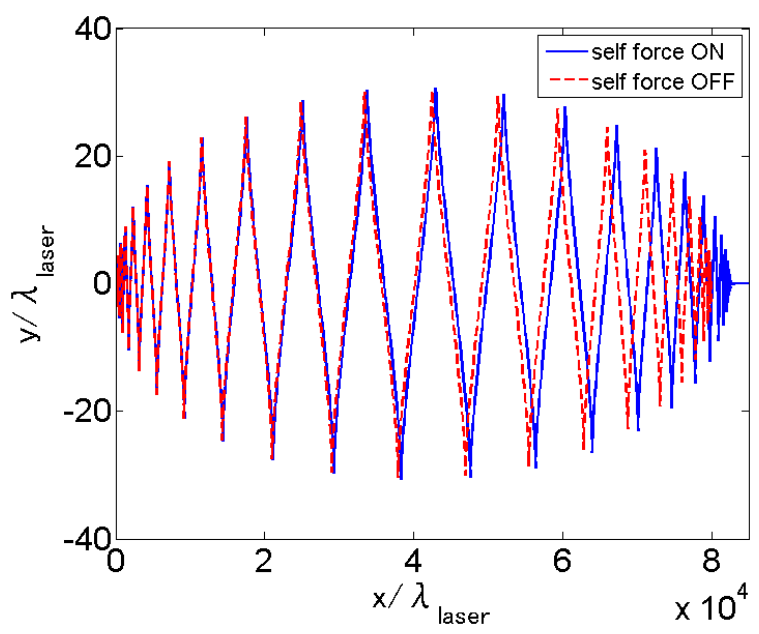

Fig. 3 The electron trajectory in $x y$ space. The electron is stationary at initial, and is accelerated by the laser field. The Laser intensity is $5 \times 10^{22} \mathrm{~W} / \mathrm{cm}^{2}$ and the wave length is $1 \mu \mathrm{m}$. The blue line is the trajectory with the reaction force and red dotted line is the one without the reaction force.

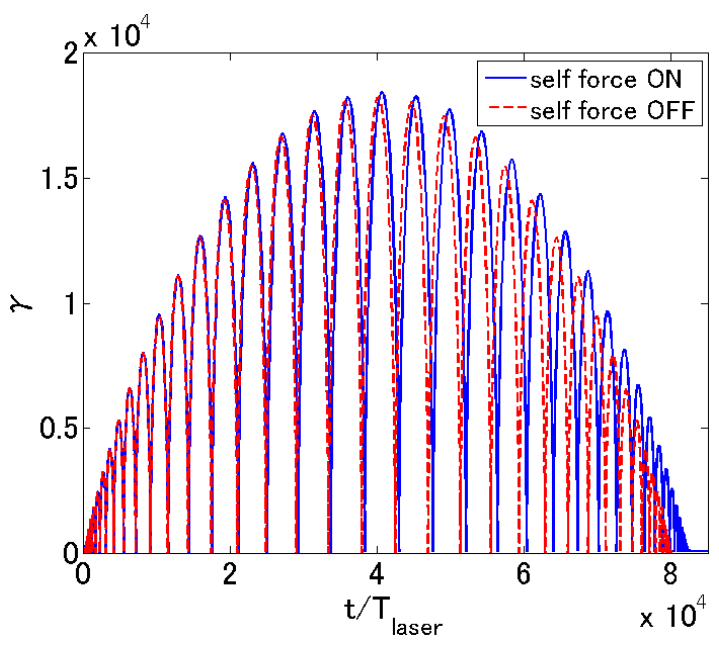

Fig. 4 Time evolution of $\gamma$. The electron is stationary at initial, and is accelerated by the laser field. The Laser intensity is $5 \times 10^{22} \mathrm{~W} / \mathrm{cm}^{2}$ and the wave length is $1 \mu \mathrm{m}$. The blue line is the trajectory with the reaction force and red dotted line is the one without the reaction force.

shown as Fig. 4. $\gamma$ is equivalent to the time element of the 4-velocity or the kinetic energy of the particle. It can be understood that there is little difference whether a reaction force is present or not from Fig. 4. This laser's normalized potential is about $a=1200$, and we often estimate the value of $\gamma$ by the following expression.

$$
\gamma=\sqrt{1+a^{2}} \approx a=1200 .
$$

However, the maximum $\gamma$ in Fig. 4 is $1.8 \times 10^{4}$. Now the laser and the electron co-move to $x$ direction, the electron is accelerated by the laser field during very long time. Therefore $\gamma$ of the electron becomes larger than estimation of Eq. (26).

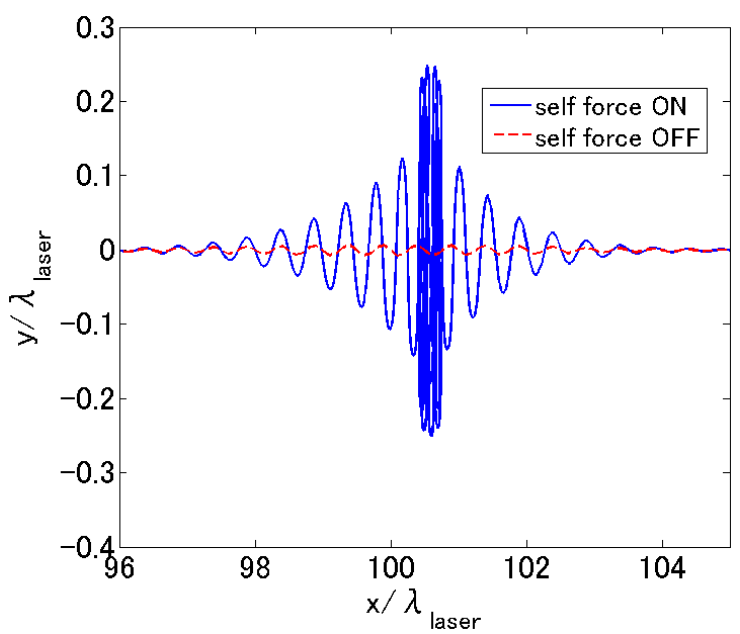

Fig. 5 The electron trajectory in $x y$ space. The electron has energy of about $1 \mathrm{GeV}$ at initial, and then the electron enter into the laser and propagate to the counter direction of the laser, from right to left. a laser intensity is $5 \times 10^{22} \mathrm{~W} / \mathrm{cm}^{2}$ and a wave length is $1 \mu \mathrm{m}$. The blue line is the trajectory with a reaction force and red dotted line is the one without a reaction force.

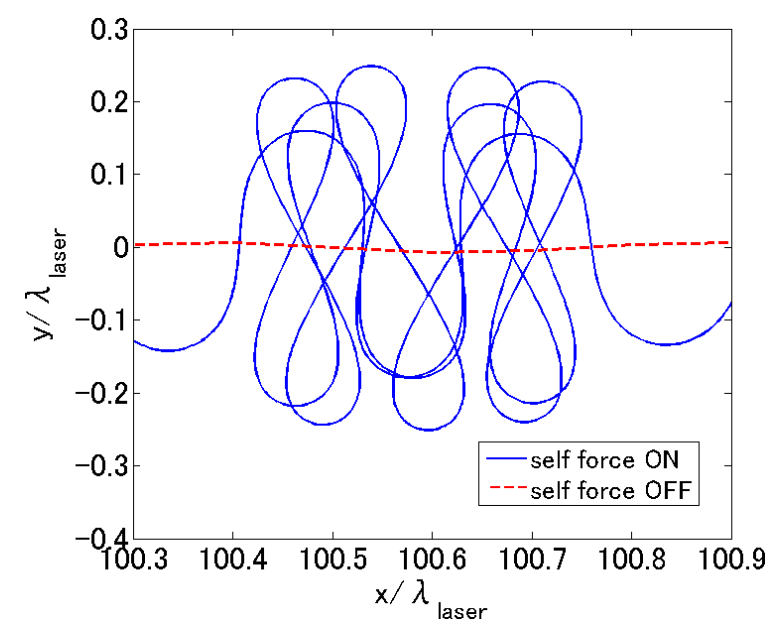

Fig. 6 The Close up figure of Fig. 5. The electron loses energy by the bremsstrahlung, and it is pushed back in the direction where the laser is propagated. The laser passes on the electron after that, and it is accelerated up to speed of light again by the Lorentz force in a rear tail part.

Next, we'll show you the result of the counter configuration. In this situation, we can observe the big effects of a radiation reaction. If the very large bremsstrahlung is caused, the particle should have big energy beforehand. When a part of the kinetic energy is discharged as bremsstrahlung, the reaction force is generated. Therefore, we need to consider that an electron with a large energy enters into the high-intense laser pulse. In connection with this simulation, J. Koga researched for this situation [6]. He calculated with a Landau-Lifshitz equation [3] as an approximation of a Lorentz-Abraham-Dirac equation. His conclusion is most of the kinetic energy is converted to the 


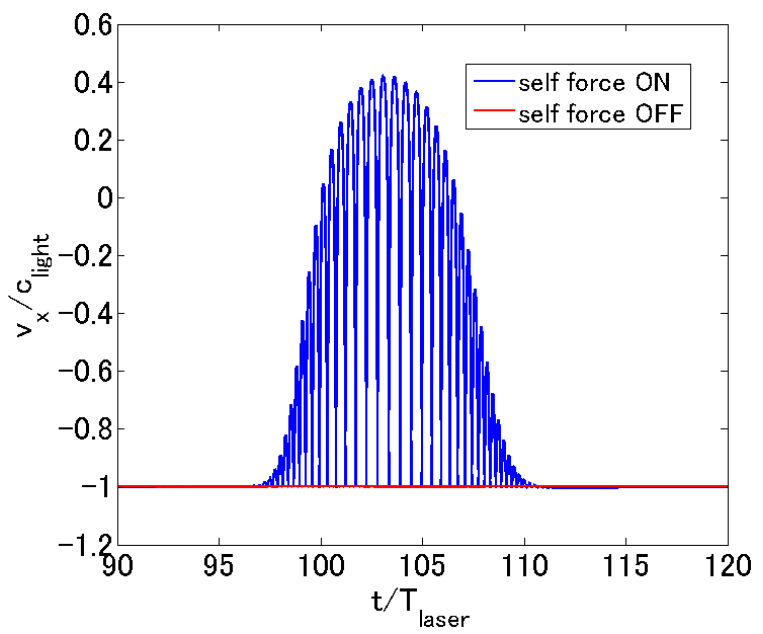

Fig. 7 The time evolution of the electron's velocity $v_{x}$. The existence of the reaction force means the kinetic energy is greatly transmutable.

energy loss as bremsstrahlung. And then, he observed a large reaction force. Our simulation can show us the result like J. Koga. The reaction effect can be checked easily from the electron's trajectory (see Fig. 5 and Fig. 6). An electron propagates from right to left in these figures. A red line in these figures is a trajectory without a reaction force. In a radiation OFF model, there isn't a mechanism of an energy loss. Therefore an initial large inertial momentum almost doesn't change. But, in a radiation ON model, the kinetic energy of an electron can be change via a bremsstrahlung. Figure 6 is close up of Fig. 5. After the electron enters into the laser, the electron is pushed back by the ponderomotive force of the ultra intense laser pulse. Of course, the direction of the ponderomotive force is opposite direction of the initial inertia of the electron. Next, the electron losing an inertia is driven by a Lorentz force as $-e \boldsymbol{v} \times \boldsymbol{B}$. When the laser passes through the electron, the ponderomotive force is generated in a rear tail of the laser. This is the reason why the electron orbit seems to be compressed. You may think the velocity changes hard from Fig. 7, however the speed of the electron is a speed of light (from Fig. 8). Figure 7 tells us that the electron takes an eight figure motion with a speed of light. The time evolution of $\gamma$ is described as Fig. 8. This figure has important information of a bremsstrahlung. The red line of a radiation OFF model shows us that the initial-inertial energy is conserved. In comparison with it, the blue line of a radiation $\mathrm{ON}$ model has a large change in the short interval of $5 \times T_{\text {laser. }}$ This change evidently means the energy loss of a radiation. The reaction force is generated with a bremsstrahlung at the same time. The information of forces is described as Fig. 9. This is information of $x$ direction, the red line is a reaction force and the blue dotted line is a Lorentz force. The order of a reaction force is same as a Lorentz force. This is one of the big reaction effects, an electron trajectory changes dramatically. We

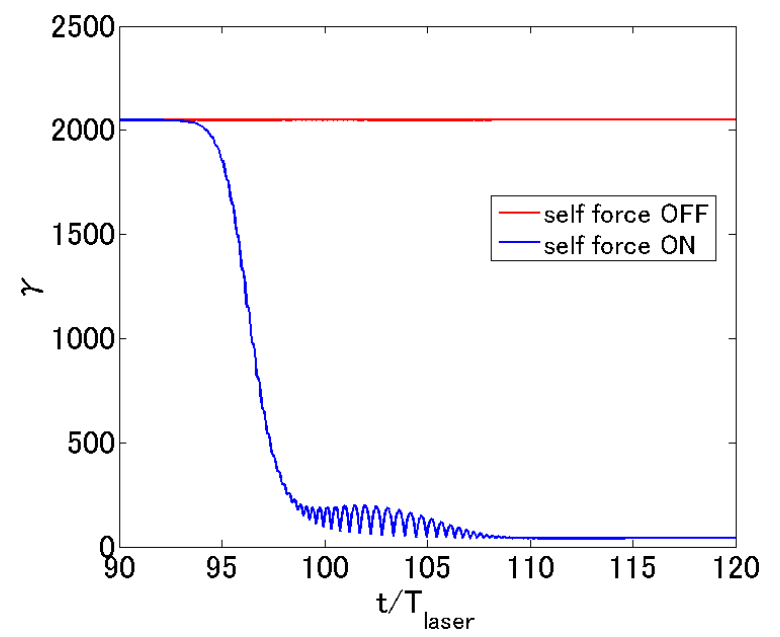

Fig. 8 The time evolution of $\gamma$ (kinetic energy). The blue line is the trajectory with the radiation force and red line is the one without the radiation force.

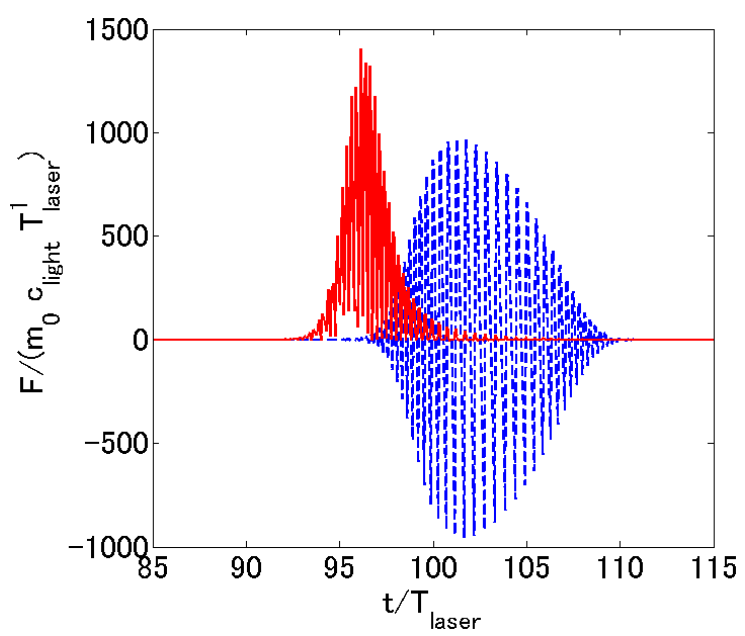

Fig. 9 The time evolution of the working force in the electron ( $x$ direction). The red line means a reaction force and the blue dotted one is a Lorentz force as $(-e \boldsymbol{v} \times \boldsymbol{B})_{x}$.

showed that a reaction force becomes large and this force affects the particle's trajectory, the energy hysteresis and the momentum.

\section{Discussion}

In an ultra-relativistic laser electron interaction, one of the most difficult problems is run-away. In solving the equation of motion (original LAD equation), we can't avoid this issue by using the theoretical solution and the simulation. A run-away effect has not to be generated in physical reality. Therefore, we want to get a correct equation of motion with a radiation reaction. In this paper, we suggested new equation which can avoid a run-away. And it is proved by theoretical calculation and simulation result. It is necessary to pay attention that our calculation result is all the same as J. Koga. In ultra-relativism, most important 
factor is $\gamma$, for that reason the highest order of $\gamma$ is effective in Eq. (20).

$$
\frac{m_{0} \tau_{0}}{c^{2}} g\left(\frac{\mathrm{d} w}{\mathrm{~d} \tau}, \frac{\mathrm{d} w}{\mathrm{~d} \tau}\right) w^{\mu}
$$

is the highest order in our equation. In fact, this term is the highest term in a Landau-Lifshitz equation too. Therefore, the simulations of us and J. Koga solved the same equation in ultra-relativism,

$$
m_{0} \frac{\mathrm{d} w^{\mu}}{\mathrm{d} \tau}=Q F_{\text {laser }}^{\mu \nu} w_{v}+\frac{m_{0} \tau_{0}}{c^{2}} g\left(\frac{\mathrm{d} w}{\mathrm{~d} \tau}, \frac{\mathrm{d} w}{\mathrm{~d} \tau}\right) w^{\mu} .
$$

In this special case, there exists a big positive value of $\gamma_{0}$ and if we roughly know the value of $\gamma$ which is satisfied $\gamma>\gamma_{0}$, and then

$$
\begin{aligned}
w^{\mu}(\tau)= & w^{\mu}(0) \times \exp \left[-\int_{0}^{\tau} \mathrm{d} \tau^{\prime} \frac{1}{m_{0} c^{2}} \frac{\mathrm{d} W}{\mathrm{~d} t}\left(\tau^{\prime}\right)\right] \\
& +\int_{0}^{\tau} \mathrm{d} \tau^{\prime} \frac{Q}{m_{0}} F_{\text {laser }}^{\mu \nu} w_{v}\left(\tau^{\prime}\right) \\
& \times \exp \left[-\int_{\tau^{\prime}}^{\tau} \mathrm{d} \tau^{\prime \prime} \frac{1}{m_{0} c^{2}} \frac{\mathrm{d} W}{\mathrm{~d} t}\left(\tau^{\prime \prime}\right)\right] .
\end{aligned}
$$

This is an analogy of Eq. (21) and Eq. (22). Strictly, a Landau-Lifshitz reaction force should be described as

$\frac{m_{0} \tau_{0}}{c^{2}} g\left(\frac{\mathrm{d} w}{\mathrm{~d} \tau}, \frac{\mathrm{d} w}{\mathrm{~d} \tau}\right) w^{\mu} \mapsto \frac{m_{0} \tau_{0}}{c^{2}} g\left(f_{\text {external }}, f_{\text {external }}\right) w^{\mu}$,

under an assumption which $\left|f_{\text {external }}^{\mu}\right| \gg\left|f_{\text {reaction }}^{\mu}\right| . \quad$ Finally, we'll show the difference between our Eq. (20) and Landau-Lifshitz equation as Fig. 10. We used normalized velocities (Newton's sense), evaluated one of Eq. (20) minus one of a Landau-Lifshitz. The simulation results seem to be the same, but a Landau-Lifshitz method has a problem. We can get the same result of Fig. 9 in LandauLifshitz method, however an assumption is broken. Saying in different words,

$\left|f_{\text {external }}^{\mu}\right| \gg\left|f_{\text {reaction }}^{\mu}\right| \approx \frac{m_{0} \tau_{0}}{c^{2}} g\left(f_{\text {external }}, f_{\text {external }}\right) w^{\mu}$,

isn't always satisfied (see Fig. 9). Therefore our method is powerful because it isn't necessary to assume any approximation. We got a great tool which can simulate all of classical laser single-electron interaction. Next our step is

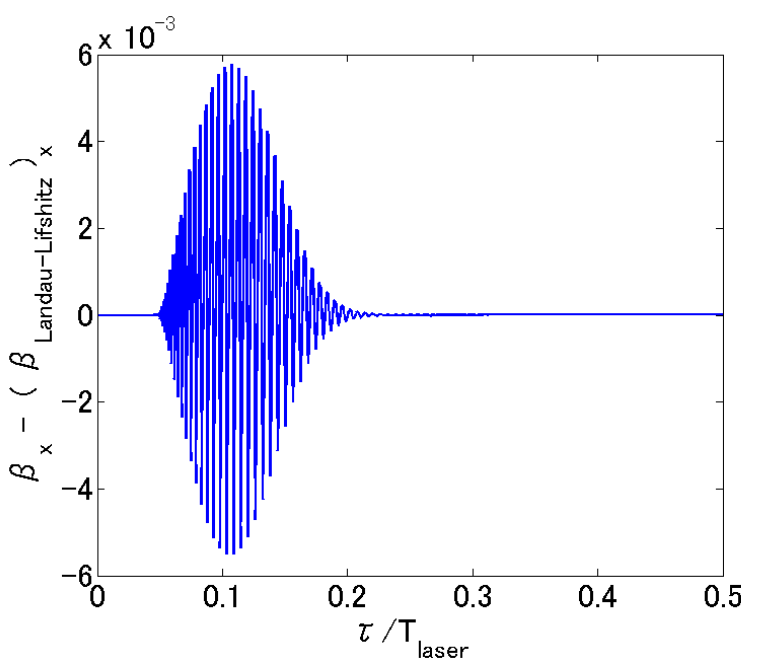

Fig. 10 The proper time evolution of the difference of the velocity. The final value of this figure becomes 0.0035 .

an expansion our simulation to multi charged particle systems.

\section{Conclusion}

We derived an equation of motion with a radiation reaction which can avoid a run-away solution without any assumption. Our equation is based on an energy balance between a work of a reaction force and an energy loss of a bremsstrahlung, as Eq. (7). The simulation result of Eq. (20) is similar output of a Landau-Lifshitz equation. And the theoretical solution shows us Eq. (20) doesn't have a run-away solution. Equation (20) is a new tool which is useful for the research of laser electron interaction with a radiation reaction.

[1] V. Yanovsky et al., Opt. Express 16, 2109 (2008).

[2] P.A.M. Dirac, Proc. Roy. Soc. A 167, 148 (1938).

[3] L.D. Landau and E.M. Lifshitz, The Classical theory of fields (Pergamon, New York, 1994).

[4] R.T. Hammond, e-print arXiv:0902.4231.

[5] R.T. Hammond, Phys. Rev. A 81, 062104 (2010).

[6] J. Koga, Phys. Rev. E 70, 046502 (2004). 